Mathematical Modelling and Analysis

Volume 5, 2000, PAGes 108-118

(C) 2000 Technika

\title{
HYBRID MODELLING IN STRATIFIED DECISION SUPPORT SYSTEMS. I
}

\author{
A. KOLESNIKOV, A. YASHIN \\ Kaliningrad State Technical University \\ Sovetsky av.1, 236000, Kaliningrad, Russia \\ S. -Petersburg Technical University \\ Politehnicheskaya 29, 195251, S. -Petersburg, Russia \\ E-mail: kolesnikov@baltnet.ru,yashin@avalon.ru
}

Received October 22, 1999

\begin{abstract}
The paper studies the basic problems of Artificial Intelligence, such as integration of difference attributes of human intellect. For this purpose we have been created synergetic systems that are hybrid intelligent systems (HYIS). The paper shows the world of decision support problems and the world of modelling approaches evolution. The term

'heterogeneous problem' for decision support systems is discussed. Two models of interaction between the problems world and the methods world also the results of HYIS creating are discussed. The formalism of HYIS is introduced.
\end{abstract}

\section{INTRODUCTION. DECISION SUPPORT SYSTEMS}

Imagine 4 different situations: the leader of a financial organization has invited experts for solving an unexpected problem; director of an enterprise at the beginning of the workday conducted a selector meeting with his division chiefs; the Board of Chief Designers has gathered in the design bureau; a state organization has attracted a Consulting Company for comprehensive analysis of the situation. In spite of differences between of these situations, they all have one feature in common: they all deal with decision support systems (DSS). Systems of the type 'Decision maker (DM) - Experts' in general used to have no proper names in the past, though they were widely used in the control and design practice, executing particular functions of assistance in solving the problems, which cannot be easily solved by one person. Only in 1970 the term of 'decision support system' appeared [26], but presently DSS is one of the 
general trends in the development of information systems. The demand for computer decision making support in Russia has noticeably risen only at the end of 1993 [23], but in 1994 it was shown by banks $(60-70 \%)$, enterprises of fuel, energy, chemical industry and state institutions. The software for the DSS on safety, engineering decision making [3], forecasting bioresources and other application domains existing today.

DSS generate alternatives, realise their evaluation, forecast consequences of the supposed decisions, choose the best variant and agree group decisionmaking [22]. N.N. Moiseev, A.A. Samarsky, G.S. Pospelov, O.I. Larichev, V.N. Burkov, E.A. Trahtengerts and other Russian scientists have significantly contributed to the DSS theory and practice. In spite of fast development of DSS and software (On-Line Analitical Processing - OLAP) for decision making support, there is still a lot of unclearness here. For instance, what kind of problems are solved by DSS? What is Integration of distributed knowledge in DSS? Which are modelling approaches and models adequate to DSS? This paper gives the answers to these questions to a certain extent.

\section{EVOLUTION OF THE WORLD OF DECISION MAKING SUPPORT PROBLEMS}

The term 'problem' derives from the Greek word 'ballein' - 'to throw', i.e. a problem is an object, which is thrown onward [10], which determines specific features of solving a problem today with a view to the future. Studying these features, we have analyzed dictionaries, encyclopedias, methodological, special mathematical, and technical literature and have selected nearly 20 definitions of the term 'problem', have studied the known classifications and have created the ontology of the problems world from 1960 till 1999. The analysis showed that little has changed in the problem specification and formalism in this period. The theoretical models are lagging behind practical needs. The problem is interpreted in the same way, as it was offered at the beginning of the 70's by the specialists on operations research R. Akof and M. Sasieni: 'It's possible to consider that a problem appears if the subject needs to reach some purpose and there are different ways to achieve this purpose. Each of them is characterized by different efficiency and moreover, there appear doubts on the choice of the best strategy' [1]. Recently the accents are being shifted very much slowly onto 'subproblems selected within a given problem' [17]. This occurs synchronously with the development and usage of expert systems (ES). This is connected with the fact that scientists are facing difficulties of solving 'not a toy problem', but "practical ones' [31] exactly in the knowledge engineering. The ontology of the problems world has responded to these processes with the terms of 'well and badly determined problems' [24], 'problems of unique choice' [9], 'simple and complex problems' [17,18], 'moderate problems' [15], 'much and specifically complex problems' [2].

At the same time, E.A. Alexandrov, studying the heuristic decision making practice [2], emphasized that such specifically complex problems appear 
everywhere, where we deal with functioning of system, depending on many heterogeneous variables. We have studied this feature of the problems of DSS taking as examples dispatcher meeting in the sea fishing port, crop capacity strategic planning by main agronomist service within a bio-production system and collective decision making in the shipyard design bureau $[4,5,28]$ from 1971 till 1999. Our results confirm that practical successes of decision making support problems modelling are associated with the perfection of the multilanguage methods [6] for presentation and manipulating of the heterogeneous state of such systems which is denominated by deterministic, stochastic, precise and fuzzy linguistical variables, as well as variable-relations (we suppose that there are certain perfect measurements of object state parameters). Direct effect of heterogeneous variables spottiness in current and target problem states is its change from the area of 'simple' to the area 'complex' problems one, which requires the principles of system analysis and syntheses.

All these features witness to the fact that in the problems world there exist objects, characteristics of which are not yet studied to the full. It causes their ambiguous understanding, using different terminology, and negative phenomena in the modelling methods world. We have introduced the 'heterogeneous problem' term in the decision support problems world in odder to indicate these objects and to study their characteristics. In this world there are no simple, complex and very complex problems. It is the world of homogeneous and heterogeneous problems. Associating the concept of 'heterogeneous' with the problem, we want to shift accents in its analysis and solution modelling onto the features determined by its internal contents and structure. Below we give a description of homo- and heterogeneous problems $(2,3)$ at the formal level, based on the conceptual model of a problem [13]:

$$
\pi=<G, D, C>
$$

where: $G$-ultimate goal (result) of problem solving; $D$ - initial data meeting the requirements of necessity and sufficiency (fullness) for obtaining the result; $C=<m, a, p>-$ conditions (known or which are still to be determined) defining more precisely the relation between $D$ and $G$ and reflecting how $D$ is transformed into $G ; m$ - the solution method (pointing it out or the description thereof); $a, p$ - the algorithm and machine program of the problem solving accordingly.

The formula (2.1) reflects a situation of abstracting from the internal essence a problem in mathematics, artificial intelligence, operation researches, and system analysis schools over the many years when they were using the idea of individual, non systematic and asocial intellect [21]. The distributed knowledge and collective efforts paradigm substituted this idea at the beginning of the 90's. This requires some revision of the term 'problem'. Below we define more precisely the formula (2.1) by introducing the term of 'homogeneous problem' which is:

$$
\pi^{h}=<G^{h}, D^{h}, C^{h}, K^{h}, O^{h}>
$$


where: $G^{h}, D^{h}, C^{h}$ - purpose, initial data and conditions of $\pi^{h}$ accordingly; $K^{h}=<{ }^{1} k_{i_{1}}^{h},{ }^{2} k_{i_{2}}^{h},{ }^{3} K^{h}>-$ classifier of the problem;

${ }^{1} k_{i_{1}}^{h} \in{ }^{1} K=\left\{{ }^{1} k_{1}, \ldots,{ }^{1} k_{8}\right\} \mid i_{1} \in \overline{1 ; 8}$ - class of the problem $\left({ }^{1} k_{1}\right.$ - 'account', ${ }^{1} k_{2}$ - 'checking', ${ }^{1} k_{3}$ - 'analysis', ${ }^{1} k_{4}$ - 'normalising', ${ }^{1} k_{5}$ - 'forecasting', ${ }^{1} k_{6}$ - 'planning', ${ }^{1} k_{7}$ - 'regulation', ${ }^{1} k_{8}$ - 'organization'); ${ }^{2} k_{i_{2}}^{h} \in{ }^{2} K=$ $\left\{{ }^{2} k_{1}, \ldots,{ }^{2} k_{5}\right\} \mid i_{2} \in \overline{1 ; 5}$ - class of variables which are necessary and sufficient to carry out manipulations for solving the $\pi^{h}$ problem ('deterministic', 'stochastic', 'fuzzy' or 'precise linguistical', 'genetic'); ${ }^{3} K^{h}=<{ }^{3} k_{q_{1}}, \ldots,{ }^{3} k_{q_{2}}>$, where: $q_{1}, q_{2}=1, \ldots, N_{3 h}, q_{2} \geq q_{1} ; \exists \pi^{h}\left({ }^{3} K^{h}=\varnothing\right),{ }^{3} K^{h}$ - the set of classes of variables-relations ('comparison', 'space', 'time', 'causality' and others $N_{3 h}$ - classes in all) which are necessary and sufficient for the manipulate ones for solving the $\pi^{h}$ problem; $O^{h}=<o_{1}^{h}, \ldots, o_{5}^{h}>-$ problem specificator; $o_{1}^{h}-$ identifier of $\pi^{h}$ (here and for other positions of the specificator, it is the LPA or the language of knowledge presentation text); $o_{2}^{h}, o_{3}^{h}-$ knowledge about the subject and reasons of $\pi^{h}$ solving; $o_{4}^{h}$ - knowledge about the relationships of $\pi^{h}$ with other homogeneous problems, falling into one and the same $\pi^{u}$ (we neglect the relationships between $\pi^{u}$ and $\left\{\pi^{h}\right\}$ ); $o_{5}^{h}$ - attribute to the heterogeneous problem $\pi^{u}$ ).

Such approach to the problem specification introduces its classification attributes, gives an opportunity to take into account personalities models [7] and information exchange while deciding the problem sets within heterogeneous problem of $\pi^{u}$, under which we will understand:

$$
\pi^{u}=<G^{u}, D^{u}, C^{u} K^{u}, O^{u}, \hat{\Pi}^{u}, R^{u h}>,
$$

where: $G^{u}, D^{u}, C^{u}$ - purpose, initial data, conditions of $\pi^{u}$ accordingly; $K^{u}$ - classifier; $K^{u}=<{ }^{1} k_{i}^{u},{ }^{2} K^{u},{ }^{3} K^{u}>\mid{ }^{1} k_{i}^{u} \in{ }^{1} K,{ }^{2} K^{u}=<{ }^{2} k_{1}, \ldots,{ }^{2} k_{f}>$ $\left|2 \leq f \leq 5,{ }^{2} k_{j} \in{ }^{2} K\right| j=1, \ldots, f,{ }^{3} K^{u}=<{ }^{3} k_{q_{3}}, \ldots,{ }^{3} k_{q_{4}}>\mid q_{3}, q_{4}=$ $1, \ldots, N_{3 u}, q_{4} \geq q_{3} ; \exists \pi^{u}\left({ }^{3} K^{u}=\varnothing\right) ; O^{u}$-specificator, $O^{u}=<o_{1}^{u}, \ldots, o_{6}^{u}, O_{7}^{u}>$ , $o_{1}^{u}$ - identificator of $\pi^{u}, o_{2}^{u}, o_{3}^{u}$ - the knowledge about DM and the causes of $\pi^{u}$ solving; $o_{4}^{u}$ - knowledge about the relationships of the given $\pi^{u}$ with other heterogeneous problems; $o_{5}^{u}$ - knowledge about the operation, executing the decision $\pi^{u} ; o_{6}^{u}$ - the evaluation of the results of the operation in the object of control; $O_{7}^{u}=\left\{o_{1}^{u}, \ldots, o_{N_{h}}^{u}\right\}$ - the set of the evaluations of results of $\pi_{1}^{h}, \ldots, \pi_{N_{h}}^{h}$ solving, $\hat{\Pi}^{u}=\left\{\hat{\pi}_{1}^{u}, \ldots, o_{N_{u}}^{u}\right\}$ - the set of decompositions; $\hat{\pi}_{i}^{u}=\left(\Pi^{h}, \hat{r}_{i}\right) \mid i \in \overline{1 ; N_{u}}$ - the decomposition of $\pi^{u}$ in the relation to $\hat{r}_{i} \in \hat{R}=\left\{\hat{r}_{1}, \ldots, \hat{r}_{N_{u}}\right\}$ - the set of the decomposition relations of $\pi^{u}$ to $\left\{\pi^{h}\right\}, \Pi^{h}=\left\{\pi_{1}^{h}, \ldots, \pi_{N_{h}}^{h}\right\}$ - the set from $N_{h}$ homogeneous problems within $\pi^{u} ; R^{u h}=\left\{{ }^{G} R^{u h},{ }^{D} R^{u h},{ }^{C} R^{u h}\right\}$ - the set of the relations of corresponding elements of $\pi^{u}$ and $\pi^{h}$.

Consider the components of $\pi^{u}$ more precisely. The purposes $G^{u}$ and $G_{1}^{h}, \ldots, G_{N_{h}}^{h}$, and the initial data $D^{u}$ and $D_{1}^{h}, \ldots, D_{N_{h}}^{h}$, can be dissimilar in the general case. For instance, $D_{1}^{h}, \ldots, D_{N_{h}}^{h}$ can be more explicated, but $D^{u}$ is more abstract). The conditions $C^{u}$ and $C_{1}^{h}, \ldots, C_{N_{h}}^{h}$ are distinguished in principle, since if for $C_{1}^{h}, \ldots, C_{N_{h}}^{h}$ we know the $m_{i}^{h}$ (otherwise the subject 
$o_{2}^{h}$ is incompetent) and $a_{i}^{h}$ and $p_{i}^{h}$ can be known, then for the $C^{u}$ the $m^{u}$ method as well as $a_{i}^{u}, p_{i}^{u}$ are the target object and the ones to be designed in HYIS.

If $N_{h}=1$, then there is the singular case of existence $\left(\pi^{u} \hat{r}_{1} \pi^{h}\right)$, where: $\hat{r}_{1}$ is the 'whole-part' relation, while $G^{u}=G^{h}, D^{u}=D^{h}, C^{u}=C^{h}, K^{u}=$ $K^{h}, O^{u}=O^{h}$ in the problem world, when the DM and the expert are the same person.

Let us suppose that in the problems world of the $S$ system there exists the $\Pi^{u}=\left\{\pi_{1}^{u}, \ldots, \pi_{N_{f}}^{u}\right\}$ finite set and $\forall \pi_{i}^{u} \exists \Pi^{h}=\left\{\pi_{1}^{h}, \ldots, \pi_{N_{h}}^{h}\right\} \mid i=$ $1, \ldots, N_{f}, \forall i\left(N_{h}=\right.$ vary $)$. Then on $\Pi^{u}$ it is possible to define the informal procedure of decomposition:

1.'start', $i=1$;

2. To take $\pi_{i}^{u} \mid i \leq N_{f}$

3. To get $\hat{\Pi}^{u}=\left\{\hat{\pi}_{1}^{u}, \ldots, \hat{\pi}_{N_{u}}^{u}\right\}$ for which (

$3.1 j=1$;

3.2. To take $\hat{r}_{j}$;

3.3. To get $\pi_{j}^{u}$ for which (

3.3.1 $n=1$;

3.3.2. To define $G_{n}^{h}, D_{n}^{h}, C_{n}^{h}, K_{n}^{h}, O_{n}^{h}$;

3.3.3. $n=n+1$ and if $k \leq N_{h}$, then go over to 3.3.2);

3.4. $j=j+1$ and if $j \leq N_{u}$, then go over to $3.2 ;$;)

4. $i=i+1$ and if $i \leq N_{f}$, then go over to 2 , otherwise - 'end'.

Finally, having regularised heterogeneous problems:

$$
W^{u}=<\Pi^{u}, R^{u}>
$$

where: $R^{u}=\left\{r_{1}^{u}, \ldots, r_{N_{w}}^{u}\right\}$ - the set of the relations on $\Pi^{u}$, for instance, 'part - whole', 'class-subclass', and others, we will complete the formation of the model $W^{u}$ of the problem world. The graphic interpretation example of $W^{u}$ (only minimum details required for understanding was indicated; dotted line shows $o_{4}^{h}$ ) is given in fig.1.

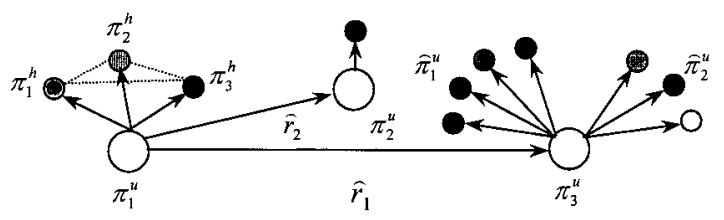

Figure 1 .

Such model avoids terminological contradictions in the world of problem ontology and explains the failure of attempts to model problems. Separate 
parts from $\pi^{u}$ 'are sliced' in these attempts, which can be imitated within the framework of a single method supported by one or another, the scientific schools. Such obviously simplified models of $\pi^{u}$, when the problem structure not taken into account (or strictly speaking, the initial problem $\pi^{u}$ has not been solved) after 'successful' laboratory tests go into practice without any prospects to be applied. DM as usual has to solve a more general (whole) $\pi^{u}$ problem and a computer solution scheme 'does not fit' into the DM creative process. Finally, $W^{u}$ possesses flexibility, and is open for modification of $\pi^{u}$ (adding and removing $\pi^{h}$ ) and the problems world as a whole (adding and removing of $\pi^{u}$ ).

\section{STRATIFIED MODELS FOR THE DSS. MULTILEVEL AP- PROACH TO SOLVING THE HETEROGENEOUS PROB- LEMS}

In 1970 s M.D. Mesarovic, D. Tako, Y. Takahara have published the 'Theory of Hierarchical, Multilevel, System' book [11] giving particular part to stratification of a system $S$. The choice of the number of strata is one of the stratification problems. If the lower limit is obvious (minimum two strata), the choice of the upper limit is dependent on the skill of the developer. We have grounded that the upper limit can require three strata, if we consider the close relation of strata and modelling methods [16] and classify them into analytical ( $A$-knowledge, $A$-methods), statistical ( $C$-knowledge, $C$-methods) and logical-linguistical ( $L$-knowledge, $L$-methods) ones. Then all the subject knowledge of experts (for instance, mechanical engineer, electrical engineer, technologist, financier, and others) are regularised into these classes and explicate them.

Let there be the system $S: X \rightarrow Y$, where: $X=X_{1} \times X_{2} \times X_{3}, Y=Y_{1} \times Y_{2} \times$ $Y_{3}$ - the input and output sets accordingly. Each couple $\left(X_{i}, Y_{i}\right) \mid i=1, \ldots, 3$ is attributed to a certain stratum $S_{i} \mid i=1, \ldots, 3$. Then it is possible to enter two models.

Model 1. The stratum $S_{i} \mid i=1, \ldots, 3$ is presented by mappings:

$$
\begin{aligned}
& S_{3}: X_{3} \times V_{3} \rightarrow Y_{3}, \\
& S_{2}: X_{2} \times B_{2} \times V_{2} \rightarrow Y_{2}, \\
& S_{1}: X_{1} \times B_{1} \rightarrow Y_{1},
\end{aligned}
$$

where: $B_{i}, V_{i}$ - the sets of influences' coming from strata adjoining $S_{i}$ from above and from below accordingly.

The family $S_{1}, S_{2}, S_{3}$ is called the $M 1$-stratification of $S$, if there exist two mapping families $\alpha_{i}: Y_{i} \rightarrow V_{i+1} \mid i=1,2$ and $\beta_{i}: Y_{i} \rightarrow B_{i-1} \mid i=2,3$, that for each $x \in X$ and $y=S(x)$ :

$$
y_{3}=S_{3}\left(x_{3}, \alpha_{2}\left(y_{2}\right)\right) \text {, }
$$




$$
\begin{aligned}
& y_{2}=S_{2}\left(x_{2}, \beta_{3}\left(y_{3}\right), \alpha_{1}\left(y_{1}\right)\right), \\
& y_{1}=S_{1}\left(x_{1}, \beta_{2}\left(y_{2}\right) .\right.
\end{aligned}
$$

It is a model of the 'distance' DSS in $S$. The $\pi^{u}$ problem of DM, which appeared at a higher abstracting level, decomposes 'downwards', but information on the solution of $\Pi^{h}$ will be sent 'upwards'.

Model 2. The stratum $S_{i} \mid i=1, \ldots, 4$ is presented by the mappings:

$$
\begin{aligned}
& \left\{S_{4}: X_{4} \times V_{i} \rightarrow Y_{4}\right\}, \\
& \left\{S_{i}: X_{i} \times B_{4} \rightarrow Y_{i}\right\}, \quad \text { for } i=1, \ldots, 3 .
\end{aligned}
$$

The $S_{1}, \ldots, S_{4}$ family is called the $M 2$-stratification of $S$, if there exist two reflection $\alpha_{i}: Y_{i} \rightarrow V_{4} \mid i=1,2,3$ and $\beta_{4}: Y_{4} \rightarrow B_{i} \mid i=2,3$, that for each $x \in X$ and $y=S(x)$ :

$$
\begin{aligned}
& y_{4}=S_{4}\left(x_{4}, \alpha_{1}\left(y_{1}\right), \alpha_{2}\left(y_{2}\right), \alpha_{3}\left(y_{3}\right),\right), \\
& y_{i}=S_{i}\left(x_{i}, \beta_{4}\left(y_{4}\right)\right) \mid i=1, \ldots, 3 .
\end{aligned}
$$

It is a model of 'personal' DSS in $S$, when DM forms the system $S^{u} \subseteq S$ for the problem $\pi^{u}$ solving, occupying the highest level $S_{4}$ in relation to expert strata $S_{i} \mid i=1, \ldots, 3$ in it where the problems $\pi^{h}$ are solved.

Let us define languages of the professional activity (LPA) sets

$L=\left\{L_{1}, \ldots, L_{n}\right\}$ in the system $S$ and the correspondence $\Phi \subseteq L \times \hat{S} \mid \Phi \neq \varnothing$, where $\hat{S}=\left\{S_{1}, S_{2}, S_{3}\right\}$ for the $M 1$-model and $\hat{S}=\left\{S_{1}, \ldots, S_{4}\right\}$ for the $M 2$ model. We assume that one stratum can be corresponded by more the one $L_{j} \in L \mid j=1, \ldots, n$. Then the 'heterogeneous application domain' is defined as follows:

$$
E^{L}=<\hat{S}, L, \Phi>\text {. }
$$

Let the heterogeneous problems $\Pi^{u}=\left\{\pi_{1}^{u}, \ldots, \pi_{N_{f}}^{u}\right\}$ also $\forall \pi_{i}^{u} \exists \Pi^{h}=$ $\left\{\pi_{1}^{h}, \ldots, \pi_{N_{h}}^{h}\right\}$, where: $i=1, \ldots, N_{f}, \forall i\left(N_{h}=\operatorname{var} y\right), \pi_{i}^{u} \in \Pi^{u}$. Let suppose that $\pi_{i}^{u}$ can appear only on $S_{i} \mid i=2,3$ and $S_{i}$ for the $M 1$ - and $M 2$ stratifications accordingly. Let us define the correspondences $\Psi_{1} \subseteq \Pi^{u} \times \hat{S}$ and $\Psi_{2} \subseteq \hat{\Pi}^{h} \times \hat{S} \mid \hat{\Pi}^{h}=\bigcup_{i}^{N_{f}} \Pi_{i}^{h}$ more then one $\pi^{h}$ can belong to $S_{i} \mid i=1, \ldots, 3$ and for the $M 2$-stratification is $\forall \pi_{4}^{u} S_{4} \in \Psi_{1} \exists^{1} \hat{\Pi}^{h} S_{1},{ }^{2} \hat{\Pi}^{h} S_{2},{ }^{3} \hat{\Pi}^{h} S_{3} \in \Psi_{2}$, where: ${ }^{1} \hat{\Pi}^{h}, \ldots,{ }^{3} \hat{\Pi}^{h} \subseteq \hat{\Pi}^{h}$ but for $M 1$-stratification $-\forall \pi_{3}^{u} S_{3} \in \Psi_{1} \exists^{1} \hat{\Pi}^{h} S_{1},{ }^{2} \hat{\Pi}^{h} S_{2} \in$ $\Psi_{2}$, and $\forall \pi_{2}^{u} S_{2} \in \Psi_{1} \exists^{1} \hat{\Pi}^{h} S_{1} \in \Psi_{2}$. Then the 'heterogeneous problem environment' is defined as follows:

$$
E^{u}=<E^{L}, \Pi^{u}, \hat{\Pi}^{h}, \Psi_{1}, \Psi_{2}>
$$

which requires a refusal of using the ready modelling (autonomous) methods of and transition to designing the method $m^{u}$ for the $\pi^{u}$ solution. 


\section{EVOLUTION OF THE WORLD OF MODELLING METHODS FOR DECISION MAKING SUPPORT PROBLEMS}

The modelling methods world is various today and it is absolutely obvious that we are dealing with a 'population' the evolution of which is still poorly studied, and the laws of progress have not yet been formulated. We have made the first step [16] in 1986, studying artificial intelligence, operations research, decision making and system analysis methods which were most frequently used in DSS and have offered a classification, grouping the methods according to their conceptual models, the language of model description and the problems solving algorithm. Such a classification motivates the 'genetic' approach to the description of the methods population and their improvement (evolution). In this approach the internal code ('genotype') is used for the method description. The code defines a place of the method in the population, the problems solved and other external features (for instance, characteristics of algorithms and programs, which are solving the problems). There appears a possibility in principle to explain such phenomena as the 'appearance of a new class of methods', the 'method's fitness', 'the development of methods outside the frameworks of autonomous methods' and others. We study the last phenomenon and progress the idea of coding and present the following autonomous method model:

$$
m^{a}=<{ }^{4} k_{i_{4}},{ }^{5} k_{i_{5}},{ }^{6} k_{i_{6}},{ }^{7} k_{i_{7}},{ }^{8} k_{i_{8}}, K^{m}, O^{m}>
$$

where: ${ }^{4} k_{i_{4}} \in{ }^{4} K=\left\{{ }^{4} k_{1},{ }^{4} k_{2},{ }^{4} k_{3}\right\} \mid i_{4} \in \overline{1 ; 5}$ - classifier of method $\left({ }^{4} k_{1}\right.$ - 'analytical', ${ }^{4} k_{2}$ - 'statistical', ${ }^{4} k_{3}$ - 'logical-linguistical' (symbolic), ${ }^{4} k_{4}$ - 'connectionist', ${ }^{4} k_{5}$ - 'evolutionary'); ${ }^{5} k_{i_{5}} \in{ }^{5} K=\left\{{ }^{5} k_{1} \ldots,{ }^{5} k_{N_{5}}\right\} \mid i_{5} \in \overline{1 ; N_{5}}-$ conceptual model ('black box', 'system of mass service', 'automatic system', 'condition-action' and others), ${ }^{6} k_{i_{6}} \in{ }^{6} K=\left\{{ }^{6} k_{1} \ldots,{ }^{6} k_{N_{6}}\right\} \mid i_{6} \in \overline{1 ; N_{6}}$ - the language of model description (equations, algorithms, rule-oriented means and others), ${ }^{7} k_{i_{7}} \in{ }^{7} K=\left\{{ }^{7} k_{1} \ldots,{ }^{7} k_{N_{7}}\right\} \mid i_{7} \in \overline{1 ; N_{7}}$ - algorithm of solving a problem (forward and backward inference in ES, forward propagation in artificial neural network (ANN), fuzzy systems (FS) reasoning, genetic algorithm (GA), methods of solving equations and others); ${ }^{8} k_{i_{8}} \in{ }^{8} K=\left\{{ }^{8} k_{1} \ldots,{ }^{8} k_{N_{7}}\right\} \mid i_{8} \in$ $\overline{1 ; N_{8}}$ - training algorithm for ANN; $K^{m}=<{ }^{9} K,{ }^{10} k_{i_{10}},{ }^{11} k_{i_{11}},{ }^{12} k_{i_{12}},{ }^{13} K>-$ classifier of problems solved by the method; $\left({ }^{9} \mathrm{~K}=<{ }^{9} k_{q_{5}} \ldots,{ }^{9} k_{q_{6}}>\mid q_{5}, q_{6}=\right.$ $1, \ldots, 7 ; q_{6} \geq q_{5}$ - the horizons of the problems solved (shift, day, decade, month, quarter, year, more year); ${ }^{i_{10}} k_{q_{10}} \in{ }^{2} K \mid i_{i_{10}} \in \overline{1 ; 5}$ - 'working' variable; ${ }^{11} k_{i_{11}} \in{ }^{11} K=\left\{{ }^{11} k_{1},{ }^{11} k_{2}\right\} i_{11}=1,2$ - the prototyped process (continuos, discrete); ${ }^{12} k_{i_{12}} \in{ }^{12} K=\left\{{ }^{12} k_{1},{ }^{12} k_{2}\right\} i_{12}=1,2$ - the number of criteria used in the problems solved (one-, mullet- criteria); ${ }^{13} \mathrm{~K}=<{ }^{13} k_{q_{1}}, \ldots,{ }^{13} k_{q_{8}}>$ $\mid q_{7}, q_{8}=1, \ldots, N_{13} ; q_{8} \geq q_{7}$ - the set of classes of problems solved (accommodation, timetables, routings, improvements and others); $O^{m}=\left\langle o_{1}^{m}, \ldots, o_{4}^{m}\right\rangle$ - method specificator; $o_{1}^{m}$ - knowledge about possible error of the solution; $o_{2}^{m}-$ knowledge about hybrid possibilities (quantitative expert evaluation of 
with what other methods can 'good' hybrids be combined); $o_{3}^{m}, o_{4}^{m}-$ knowledge about method advantages and disadvantages accordingly (in the form of mappings onto the scales of 'good - bad' work with noise, 'good - bad' adaptability).

The autonomous methods $m^{a}$ are used for developing the autonomous models $\dot{m}^{a}$ algorithms $a^{a}$ and programs $p^{a}$. They form $W^{a}$.

The variety in $W^{a}$ for solving the DSS problems always worried the scientists. Attempts to make this variety uniform [3] and attempts to integrate continuous and discrete components [25] did not leave mathematical modelling frameworks and are used for narrow classes of control systems. The idea of mixing (combining) $m^{a}$ in synergetic systems $[4,14,20,21,27-30]$ appeared to be more fruitful. It defines evolution processes in $W^{a}$ to a certain extent: the selection of methods-parents, combining $m^{a}$ on a 'genetic' level, and the choice for the hybrid-descendant of characteristics identical to heterogeneous properties of $\pi^{u}$. So, for instance, ES, probably were the first to deal with $\pi^{u}$ and begun to evolve from classical resolutions to the real world heuristics. Today it is not possible to find a 'clean, autonomous' commercial ES shell, but their hybrid are broadly offered with single-line programming, fuzzy logic and the traditional information technologies.

The typical picture of the modelling methods world of the 80 's [8] is shown in fig. 2 a). The narrow areas of general problems are characteristic of it. The asymmetry and non-systematic character of each approach are seen in autonomous $m^{a}$. The integration area is indicated by the grey circle.

The other, a little idealised, situation of the 90's is shown in fig. $2 \mathrm{~b}$ ).
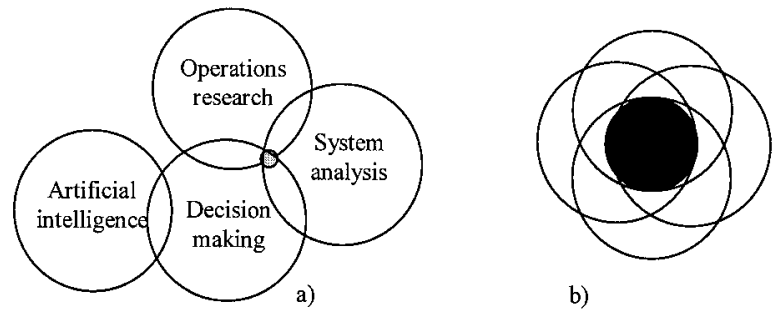

b)

Figure 2.

The difference of pictures cab be explained by the fact that more and more developers are willing to solve $\pi^{u}$ which influences $W^{a}$. This forces scientists to search for models outside the frameworks of individual schools, this leads to greater symmetries, centripetal trends, to a more systematic approach to the extension of the area modelling methods integration.

Mixed, heterogeneous, cognitive structures from $A$-, $C$ - and $L$ - knowledge are formed and kept in subject's memory in the course of his educating at all the education system stages and practical cognition of processes in large systems. This is also confirmed by the LPA analysis results, where there is a 
'mixture' of analytical dependencies presented in formulas or charts, statistic tables and linguistical (precise and fuzzy) variables, as well as the results of talks with experts who, explaining a problem, 'switch' on to different reasoning schemes - calculations, statistical conclusions and logical argumentation, manipulating graphic imagesis. All of this shows that the list of 'Nonfactors' which was introduced by A.S. Narinjani [12], can be increased by non-homogeneity of problem solving and non-homogeneity of reasoning.

\section{REFERENCES}

[1] R.Ackoff, M.Sasieni. Fundamentals of Operation Research. Mir, M., 1971. (in Russian)

[2] E.A.Alexandrov. Fundamentals of Heuristics Solving Theory. Sov. Radio, M., 1975. (in Russian)

[3] N.P.Buslenko. Complex Systems Modeling. Nauka, M., 1978. (in Russian)

[4] Ja.A.Gelfandbeyn, A.V.Kolesnikov, I.D.Rudinsky. Hybrid Modeling of Transport Node is using this Kind of Modeling for Decision-Making. In: The Decision Making Methods and Systems, Riga, 1984.(in Russian)

[5] A.V.Kolesnikov, P.M.Klachek. Intelligent Decision Support System for Agricultural Cultures Cultivate. In: International Conf. on Soft Computing and Measurements SCM 98, V.2, Sankt-Petersburg, 1998, 227-231.(in Russian)

[6] A.V.Kolesnikov. Multilanguage Models and Modeling. In: The Decision -Making Methods and Systems. Expert Systems for Aided Design, Politechnical University of Riga, Riga, 1990, 97-101.(in Russian)

[7] A.V.Kudakov, A.V.Rechinsky, A.V.Schukin, A.M.Yashin. The Mathematical Fundaments of Artificial Intelligence. Part 1. The Expert Systems. SPbGTU, SanktPetersburg, 1998. (in Russian)

[8] O.I.Larichev. Objective Models and Subjective Solutions. Nauka, M., 1987. (in Russian)

[9] O.I.Larichev. Decision Making Science and Creative work. Nauka. M., 1979. (in Russian)

[10] Ja. -L.Lorijer. The Artificial Intelligence Systems. Mir, M., 1991. (in Russian)

[11] M.D.Mesarovic, D.Macko, Y.Takahara. Theory of Hierarchical, Multilevel, Systems. Mir, M., 1973. (in Russian)

[12] A.S.Narinjani. Non-factors and Knowledge Engineering: from Naive Formalizing to Natural Pragmatic. In: Pr. of the 'Artificial Intelligence-94 (CAI'94) Conference, Rubinsk, 1994, 3-18.(in Russian)

[13] V.V.Nechaev. Creative Problem and Intelligent Solver: Conceptual Approach. In: Knowledge Presentation Models and Systems, MIREA, M., 1990, 53-68.(in Russian)

[14] G.S.Osipov. Dynamic in Systems, which are based on Knowledge. The Theory and Control Systems, (5), 1998, 24-28.(in Russian)

[15] M.Peshel. Signals and Systems Modeling. Mir, M., 1981. (in Russian)

[16] Multilanguage Semiotic Models for Mathematical Support of ASU, ASUTP and $S A P R$ for Fishing Industry. Research Report 'Information, Mathematical and Software Support of CAM and CAD for Fishing Industry'. The N of State Registration is 01860015101, the Inventory N is 02860109459, Kaliningrad, 1986. (in Russian)

[17] E.V.Popov, I.B.Fominyh. E.B.Kisel, M.D.Shapot. Statically and Dynamical Expert Systems. Finansy i Statistika, M., 1996. (in Russian)

[18] Knowledge Presentation and Usage. Trans. from Japanese/edit. by Ueno X., Isudzuka, Mir, M., 1989. (in Russian) 
[19] G.V.Rybina. Automation of Knowledge Bases Development for Integrated Expert Systems. The Theory and Control Systems, (5), 1998, 52-166.(in Russian)

[20] V.B.Tarasov. Artificial Live and Fuzzy Evolution Multi-Agent Systems - Basic Theoretical Approach to Intelligent Organization Creation. The Theory and Control Systems, (5), 1998, 12-23.(in Russian)

[21] E.A.Trahtengerts. Computer Analyses in Decision-Making Dynamic. The Devices and Systems of Control, (1), 1997, 49-56 .(in Russian)

[22] V.Shershulsky. Russian Market of Decision Support Systems. The Open Systems, (18), 1994, 1, 54-60 .(in Russian)

[23] A.M.Andrew. Artificial Intelligence. Mir, M., 1985. (in Russian)

[24] M.S.Branicky, V.S.Bonkar, S.K.Mitter. A Unified Framework for Hybrid Control: Background, Model and Theory. In: Pr. of The 33rd IEEE Conference on Decision and Control, Lake Buena Vista, 1994.

[25] S.B.Eom. Decision Support Systems Research: Reference Disciplines and a Cumulative Tradition. The International Journal of Management Science, 23 (5), Oct.1995, 511523.

[26] S.Goonatilake, S.Khebbal (Eds.). Intelligent Hybrid Systems. John Wiley \& Sons, 1995.

[27] A.Kolesnikov. Computer Aided Design of Hybrid Models for Automation Ship Systems. In: Proc. of the Conference AUTOMATION' 99, Konferencja Naukowo-Techniczna AUTOMATION' 99, Warszawa, 1999, 281-285.

[28] L.R.Medsker. Hybrid Neural Network and Expert Systems. Kluwer Academic Publishers, Boston/Dordrecht /London, 1994.

[29] L.R.Medsker. Hybrid Intelligent Systems. Internation Jornal of Computation Intelligence and Organizations, 1 (1), 1996, 10-20.

[30] S.J.Russell, P.Norvig. Artificial Intelligence. A Modern Approach. Prentice Hall, 1995.

\section{HIBRIDINIS SPRENDINIU PRIE்MIMO SISTEMŲ MODELIAVIMAS. I.}

\section{A. KOLESNIKOV, A. YASHIN}

Straipsnyje nagrinėjamos pagrindinès dirbtinio intelekto problemos, tame tarpe žmogaus intelekto savybiu integravimas. Tuo tikslu yra sukurtos hibridinès protingos sistemos. Straipsnyje analizuojamos sprendinių prièmimo sistemos ir modelinių artinių vystymasis. Aptariamas sprendinių prièmimo sistemų heterogeniškumas. Apibrēžtas hibridinių protingu sistemu formalizmas. 